\title{
Loop corrections to pion and kaon neutrinoproduction
}

\section{Marat Siddikov*, Ivan Schmidt}

Departamento de Física, Universidad Técnica Federico Santa María, y Centro Científico - Tecnológico de Valparaíso, Casilla 110-V, Valparaíso, Chile

We present our results on study of the next-to-leading order corrections to deeply virtual light meson production in neutrino experiments. We found that in the kinematics of the MINERVA experiment at FERMILAB they increase the leading order cross-section by up to a factor of two and for this reason should be taken into account in the evaluations of all observables.

The European Physical Society Conference on High Energy Physics

5-12 July, 2017

Venice

${ }^{*}$ Speaker. 


\section{Introduction}

The generalized parton distributions (GPDs) are used today for concise description of the nonperturbative structure of the target. The collinear factorization theorems [1,2] relate the GPDs to the cross-section of physical processes in Bjorken kinematics, allowing to access them experimentally. In the early studies the GPDs were extracted from experimental data on deeply virtual Compton scattering (DVCS) and deeply virtual meson production (DVMP), however it was soon realized that due to the rich structure of GPDs, additional channels were needed.

Earlier [3] we suggested that GPDs could be studied in neutrino-induced deeply virtual meson production ( $v$ DVMP) of the pseudo-Goldstone mesons $(\pi, K, \eta)$, using the high-intensity NuMI beam at Fermilab. For studies of flavor structure, $v$ DVMP has a clear advantage compared to electroproduction: the axial part of the weak current probes large contributions from the unpolarized GPDs, $H, E$ and has smaller contamination by twist-three corrections [4]. In view of the large potential of $v$ DVMP for proton GPD studies, we extend the analysis of the process and in this proceeding present results for the next-to-leading order (NLO) corrections to the $v$ DVMP amplitude. As we will show below, for the kinematics of ongoing and forthcoming neutrino experiments, where typical virtualities $Q^{2}$ are not very large, the NLO corrections are significant and for this reason should be taken into account in the analysis of experimental data.

The paper is organized as follows. In Section 2 we briefly describe the framework used for evaluation of the light meson production by neutrinos (a more detailed description might be found in our [5]). In Section 3 we present numerical results and draw conclusions.

\section{Cross-section of the $v$ DVMP process}

The cross-section of the light pseudoscalar meson production in the neutrino-hadron collisions has a form

$$
\frac{d \sigma}{d t d x_{B} d Q^{2}}=\Gamma \sum_{v v^{\prime}} \mathscr{A}_{v^{\prime}, v}^{*} \mathscr{A}_{v^{\prime}, v}
$$

where we use standard notations $Q^{2}=-q^{2}$ for the virtuality of the charged boson, $x_{B}=Q^{2} /(2 p \cdot q)$ for the Bjorken variable, $t=\left(p_{2}-p_{1}\right)^{2}$ for the momentum transfer, and the subscript indices $v$ and $v^{\prime}$ in the amplitude $\mathscr{A}$ refer to helicity states of the baryon before and after interaction. The kinematic factor $\Gamma$ in (2.1) is different for charged current and neutral current processes and is given explicitly by

$$
\Gamma_{C C}=\frac{G_{F}^{2} f_{M}^{2} x_{B}^{2}\left(1-y-\frac{\gamma^{2} y^{2}}{4}\right)}{64 \pi^{3} Q^{2}\left(1+Q^{2} / M_{W}^{2}\right)^{2}\left(1+\gamma^{2}\right)^{3 / 2}}, \quad \Gamma_{N C}=\frac{\Gamma_{C C}}{\cos ^{4} \theta_{W}} \frac{\left(1+Q^{2} / M_{W}^{2}\right)^{2}}{\left(1+Q^{2} / M_{Z}^{2}\right)^{2}},
$$

where $\theta_{W}$ is the Weinberg angle, $M_{W}$ and $M_{Z}$ are the masses of the heavy bosons $W^{ \pm}$and $Z$, $G_{F}$ is the Fermi constant, $f_{M}$ is the produced meson (pion or kaon) decay constant, and we have introduced the shorthand notations

$$
\gamma=\frac{2 m_{N} x_{B}}{Q}, \quad y=\frac{Q^{2}}{s_{v p} x_{B}}=\frac{Q^{2}}{2 m_{N} E_{v} x_{B}},
$$


where $E_{V}$ is the neutrino energy in the target rest frame. Due to the factorization theorem, the amplitude $\mathscr{A}_{v^{\prime}, v L}$ in (2.1) may be presented as a convolution of hard and soft parts,

$$
\mathscr{A}_{v^{\prime}, v}=\int_{-1}^{+1} d x \sum_{q=u, d, s \lambda \lambda^{\prime}} \sum_{v^{\prime}, v}^{q} \mathscr{C}^{q}
$$

where $x$ is the average light-cone fraction of the parton, $\lambda, q\left(\lambda^{\prime}, q^{\prime}\right)$ are the corresponding helicity and flavor of the initial (final) partons, and $\mathscr{C}_{\lambda^{\prime} v^{\prime}, \lambda v}^{q}$ is the process dependent hard coefficient function evaluated in perturbative QCD. The full expression in the next-to-leading order might be found in [5], here we omit it due to space limitations. The soft matrix element $\mathscr{H}_{v^{\prime} \lambda^{\prime}, v \lambda}^{q}$ in (2.4) is related to the leading twist GPDs as

$$
\begin{aligned}
\mathscr{H}_{v^{\prime} \lambda^{\prime}, v \lambda}^{q^{\prime} q} & =\frac{2 \delta_{\lambda \lambda^{\prime}}}{\sqrt{1-\xi^{2}}}\left(\begin{array}{cc}
-g_{A}^{q}\left(\begin{array}{cc}
\left(1-\xi^{2}\right) H^{q^{\prime} q}-\xi^{2} E^{q^{\prime} q} & \frac{\left(\Delta_{1}+i \Delta_{2}\right) E^{q^{\prime} q}}{2 m} \\
-\frac{\left(\Delta_{1}-i \Delta_{2}\right) E^{q^{\prime} q}}{2 m} & \left(1-\xi^{2}\right) H^{q^{\prime} q}-\xi^{2} E^{q^{\prime} q}
\end{array}\right)_{v^{\prime} v} \\
& +\operatorname{sgn}(\lambda) g_{V}^{q}\left(\begin{array}{cc}
-\left(1-\xi^{2}\right) \tilde{H}^{q^{\prime} q}+\xi^{2} \tilde{E}^{q^{\prime} q} & \frac{\left(\Delta_{1}+i \Delta_{2}\right) \xi \tilde{E}^{q^{\prime} q}}{2 m} \\
\frac{\left(\Delta_{1}-i \Delta_{2}\right) \xi \tilde{E}^{q^{\prime} q}}{2 m} & \left(1-\xi^{2}\right) \tilde{H}^{q^{\prime} q}-\xi^{2} \tilde{E}^{q^{\prime} q}
\end{array}\right)_{v^{\prime} v}
\end{array}\right),
\end{aligned}
$$

where the skewness $\xi$ is defined as $\xi=-\Delta^{+} / 2 \bar{P}^{+} \approx x_{B} /\left(2-x_{B}\right)$, and the constants $g_{V}^{q}, g_{A}^{q}$ are the vector and axial current couplings to quarks.

\section{Numerical results and discussion}

For the sake of definiteness, for numerical estimates we use the Kroll-Goloskokov parametrization of GPDs [6]. Using for reference the kinematics of MINERvA experiment [7], we assume that the energy of the neutrino beam is $E_{v}=6 \mathrm{GeV}$. The fact that the beam is not monochomatic leads to only mild smearing of the predicted cross-sections.

In the Figure 1 we illustrate the predictions for the cross-section of charged and neutral pion production on protons and neutrons. For all cross-sections, at fixed virtuality $Q^{2}$ we have a similar bump-like shape, which is explained by a competition of two factors. At small $x_{B} \sim Q^{2} / 2 m_{N} E_{V}$ the elasticity $y$ defined in (2.3) approaches one, for this reason the prefactor in (2.1) causes a suppression at small $x$. In the limit $x \rightarrow 1$, a suppression $\sim(1-x)^{n}$, where $n \gtrsim 3$, is due to the parton distributions. As we can see from a comparison of the leading order (dashed lines) and the full result (solid line surrounded by the green band), the next-to-leading order corrections are large. The width of the error band reflects the uncertainty due to omitted higher order loop corrections, which has been roughly estimated varying the scale in the range $\mu_{F} \in(Q / 2,2 Q)[8,9,10]$. In average the NLO correction increases the cross-section by approximately 30-50 per cent. In the special case of the charged current $\pi^{+}$production on protons, the effect of NLO correction is considerably larger. This happens because, in the leading order there is a partial cancellation of the $s$-channel and $u$-channel handbag contributions, which leads to a relatively small cross-section compared to the same process on neutrons [3]. In the next-to-leading order, such cancellation is less complete, for this reason loop corrections are more pronounced. Similarly, loop corrections are large in the case of strangeness production (kaons, $\Sigma / \Lambda$ baryons), we refer the reader to [5] for more detailed discussion. 

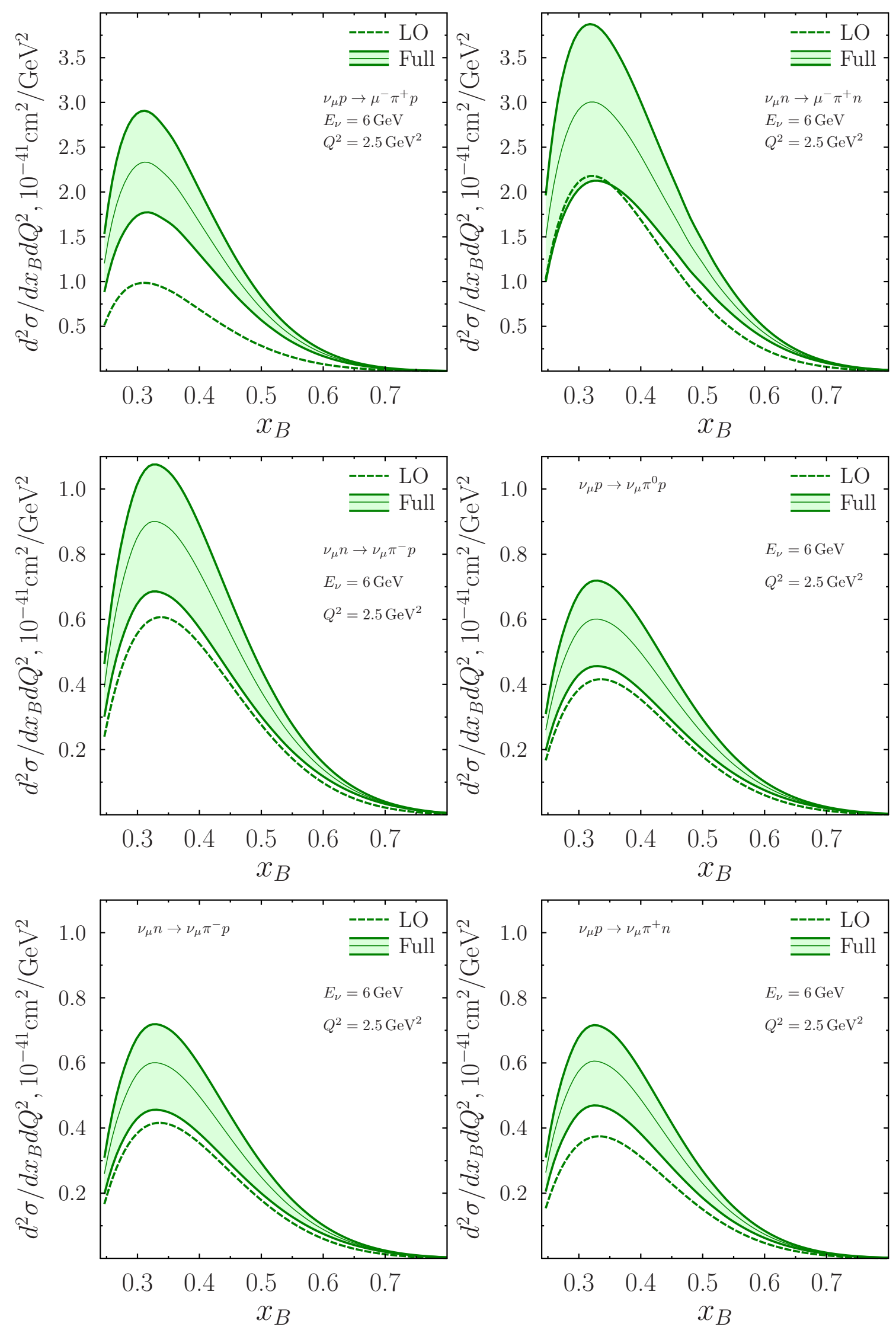

3

Figure 1: (color online) Pion production on nucleons, with neutral and charged currents at fixed energy neutrino beam $\left(E_{v} \approx 6 \mathrm{GeV}\right)$. The dashed line stands for the leading order evaluation, whereas the solid line surrounded by green error bands (marked as "Full") stands for the full result with NLO corrections. The width of the band represents the uncertainty due to the factorization scale choice $\mu_{F} \in(Q / 2,2 Q)$, as explained in the text. 
To summarize, we estimated the contributions of the next-to-leading order corrections to light meson production in the kinematics of the MINERVA experiment at FERMILAB and the planned Muon Collider/Neutrino Factory [11]. We found that these corrections are sizeable and for this reason should be included in the analysis of experimental data. Qualitatively, our findings agree with large NLO corrections to meson electroproduction $[9,10]$, deeply virtual Compton scattering [12,13] and timelike Compton scattering [14] in electron-induced processes. In view of this result, a future analysis of the next-to-next-to-leading order corrections is desirable.

\section{Acknowledgments}

This research was partially supported by Proyecto Basal FB 0821 (Chile), the Fondecyt (Chile) grants 1140390 and 1140377, and CONICYT (Chile) grant PIA ACT1413.

Powered@NLHPC: This research was partially supported by the supercomputing infrastructure of the NLHPC (ECM-02). Also, we thank Yuri Ivanov for technical support of the USM HPC cluster where part of evaluations were done.

\section{References}

[1] X. D. Ji and J. Osborne, Phys. Rev. D 58 (1998) 094018 [arXiv:hep-ph/9801260].

[2] J. C. Collins and A. Freund, Phys. Rev. D 59, 074009 (1999).

[3] B. Z. Kopeliovich, I. Schmidt and M. Siddikov, Phys. Rev. D 86 (2012), 113018 [arXiv:1210.4825 [hep-ph]].

[4] B. Z. Kopeliovich, I. Schmidt and M. Siddikov, Phys. Rev. D 89, no. 5, 053001 (2014) [arXiv:1401.1547 [hep-ph]].

[5] M. Siddikov and I. Schmidt, Phys. Rev. D 95, no. 1, 013004 (2017) [arXiv:1611.07294 [hep-ph]].

[6] S. V. Goloskokov and P. Kroll, Eur. Phys. J. A 47, 112 (2011) [arXiv:1106.4897 [hep-ph]].

[7] D. Drakoulakos et al. [Minerva Collaboration], hep-ex/0405002.

[8] A. V. Belitsky and D. Mueller, Phys. Lett. B 513, 349 (2001) [hep-ph/0105046].

[9] D. Y. Ivanov, L. Szymanowski and G. Krasnikov, JETP Lett. 80, 226 (2004) [Pisma Zh. Eksp. Teor. Fiz. 80, 255 (2004)] Erratum: [JETP Lett. 101, no. 12, 844 (2015)], [hep-ph/0407207].

[10] M. Diehl and W. Kugler, Eur. Phys. J. C 52, 933 (2007) [arXiv:0708.1121 [hep-ph]].

[11] M. M. Alsharoa et al. [Muon Collider/Neutrino Factory Collaboration], Phys. Rev. ST Accel. Beams 6 (2003) 081001 [hep-ex/0207031].

[12] A. V. Belitsky, D. Mueller, L. Niedermeier and A. Schafer, Phys. Lett. B 474, 163 (2000) [hep-ph/9908337].

[13] A. V. Belitsky and D. Mueller, Phys. Lett. B 417, 129 (1998) [hep-ph/9709379].

[14] B. Pire, L. Szymanowski and J. Wagner, Phys. Rev. D 83, 034009 (2011) [arXiv:1101.0555 [hep-ph]]. 